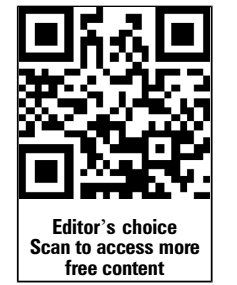

${ }^{1}$ Department of Ophthalmology, Eugene and Marilyn Glick Eye Institute, Indianapolis, Indiana, USA ${ }^{2}$ Department of Mathematical Sciences, Indiana UniversityPurdue University at Indianapolis, Indianapolis, Indiana, USA

\section{Correspondence to} Professor Alon Harris, Department of Ophthalmology, Eugene and Marilyn Glick Eye Institute, Indiana University School of Medicine, 1160 W. Michigan St., Indianapolis, IN 46202, USA

alharris@indiana.edu

Received 20 June 2013 Revised 25 September 2013 Accepted 13 November 2013 Published Online First 4 December 2013

\title{
Cerebrospinal fluid pressure and glaucoma: regulation of trans-lamina cribrosa pressure
}

\author{
Brian Marek, ${ }^{1}$ Alon Harris, ${ }^{1}$ Priyanka Kanakamedala, ${ }^{1}$ Eric Lee, ${ }^{1}$ \\ Annahita Amireskandari, ${ }^{1}$ Lucia Carichino, ${ }^{2}$ Giovanna Guidoboni, ${ }_{1}^{1,2}$ \\ Leslie Abrams Tobe, ${ }^{1}$ Brent Siesky ${ }^{1}$
}

\section{ABSTRACT}

Increased trans-lamina cribrosa pressure difference (TLCPD), the difference of intraocular pressure (IOP) and orbital cerebrospinal fluid pressure (CSF-P), has been investigated as a possible risk factor in glaucoma pathogenesis. In fact, lower CSF-P in the setting of normal IOP has been implicated as a potential risk factor for normal tension glaucoma. Increased TLCPD has been associated with decreased neuroretinal rim area and increased visual field defects. Furthermore, dysregulation of systemic blood pressure has been associated with changes in IOP. Recent studies have also suggested that increased body mass index (BMI) is associated with decreased prevalence of glaucoma, which may be due to an increased CSF-P with increased BMI found in many studies. Given the interaction of various pressures, their role in glaucoma pathophysiology has come under investigation and warrants further study in order to better understand the aetiology and progression of glaucoma.

\section{INTRODUCTION}

Glaucoma is a multifactorial disease resulting in progressive loss of retinal nerve ganglion cells, as evidenced by worsening visual fields and increased cup-to-disc ratios. ${ }^{1}$ It is the second leading cause of blindness worldwide. ${ }^{2}$ In fact, one study estimated that over 79.6 million people would have glaucoma worldwide by 2020 , of whom $74 \%$ would develop open angle glaucoma (OAG). ${ }^{3}$ Additionally, the global disability adjusted life years, a measure of overall disease burden, of glaucoma has risen from 443000 years in 1990 to 943000 years in $2010 .^{45}$ Despite its prevalence, the aetiology of glaucoma remains unclear. Various studies have documented the existence of elevated IOP in patients with and without glaucomatous damage, leading to division of patients into OAG and ocular hypertensive (OHT) groups, respectively. The existence of OHT has increased investigation of various pressures that may be implicated in glaucoma. This review will discuss the interactions of various pressures that may contribute to glaucoma: intraocular pressure (IOP), blood pressure (BP) and cerebrospinal fluid pressure (CSF-P). Special focus will be placed on the relationship between CSF-P and IOP and how these two forces contribute to glaucoma pathophysiology by creating a trans-lamina cribrosa pressure difference (TLCPD). A literature search of online databases PubMed and Medline was conducted using key term glaucoma along with cerebrospinal fluid pressure, intracranial pressure and trans-lamina cribrosa pressure. References from articles were also considered to ensure completeness.

\section{IOP, CSF-P AND TLCPD}

The forces of IOP and CSF-P meet at the lamina cribrosa, a modified extension of the peripapillary scleral flange, composed of collagen and noncollagen components. ${ }^{6}$ The lamina cribrosa forms a barrier between the intraocular space and the retrobulbar space. ${ }^{7}$ It functions as a barrier between the posterior force of the IOP and the anterior force of the CSF-P within the orbit, also known as the TLCPD (TLCPD =IOP-CSF-P). Studies have shown that the retrolaminar tissue pressure is $4 \mathrm{mg} \mathrm{Hg}$ when CSF-P is $0 \mathrm{~mm} \mathrm{Hg} .^{8}$ The ability of the lamina cribrosa to withstand the pressure gradient without deforming is dependent on its thickness, the rigidity of the extracellular matrix and the peripheral scleral tension. The lamina cribrosa's ability to maintain shape is important in protecting the structures that pass through it: the retinal ganglion cell axons, the central retinal artery (CRA) and the central retinal vein. Increased TLCPD could cause bowing of the lamina cribrosa. Such deformity may damage optic nerve ganglion cells via mechanical compression or ischaemia as the vessels pass through the lamina cribrosa. $^{9}$

While the effect of position on pressures will be discussed in detail later, it is important to note that one study found average IOP in a healthy patient population to be $14.3 \pm 2.6 \mathrm{~mm} \mathrm{Hg}$, while average CSF-P in the lateral decubitus position was found to be $12.9 \pm 1.9 \mathrm{~mm} \mathrm{Hg} .{ }^{10}$ In a lateral decubitus position, assuming no obstruction to CSF flow, there will be a posterior force on the lamina cribrosa. TLCPD could increase further if an individual had increased IOP and/or decreased CSF-P. To this end, studies have addressed the relationship between CSF-P and glaucoma.

Yablonski and colleagues evaluated the TLCPD in cats by lowering the intracranial pressure (ICP) and by lowering IOP in only one eye. ${ }^{11}$ After 3 weeks, histological examination of the optic nerve heads of eyes with unaltered IOP revealed prelaminar axonal swelling, optic disc cup enlargement and posterior displacement of the lamina cribrosa. When IOP was lowered with ICP, thus minimising alterations in TLCPD, no ocular changes were found. ${ }^{11}$

\section{LAMINA CRIBROSA STRUCTURE}

Lamina cribrosa structure provides additional insight into mechanisms of glaucomatous damage. 
The lamina cribrosa allows retinal ganglion cell axons to exit the eye through 500-600 pores. ${ }^{6}$ These pores are of differing diameter and depth depending on location within the disc-like structure. The pores in the superior and inferior portions of the lamina cribrosa are larger and contain a greater number of nerve fibres. ${ }^{6}$ The optic nerve is nourished by capillaries within the lamina cribrosa, which is supplied by the short posterior ciliary arteries. ${ }^{12}$ The superior and inferior portions are also where damage from glaucoma first occurs. Since less connective tissue exists between these pores to provide structural and nutritional support, the fibres may be more susceptible to mechanical or vascular changes from an increased pressure gradient. ${ }^{6}$

Recently, mathematical models have been developed to model the effect of lamina cribrosa deformation on CRA blood flow. ${ }^{9}$ It is hypothesised that posterior displacement of the lamina cribrosa deforms the CRA and consequently decreases blood flow. The CRA blood flow velocities were calculated with the mathematical models as a function of IOP. The latter affected lamina cribrosa displacement according to the elastic properties provided by the model. This model mirrors data previously obtained utilizing colour Doppler imaging (CDI) measurements of CRA peak systolic velocity as IOP was experimentally increased with suction ophthalmodynamometry. ${ }^{9}$ With further development of these models, a patient's risk for glaucoma progression based on various ocular measurements and vascular parameters can potentially be better evaluated.

Noteworthy in this discussion of TLCPD, however, is that CSF occupies a fluid-filled compartment that changes position within space and is subject to gravity. Therefore, the pressure exerted by CSF varies with position of the area in question relative to the vertical position of the whole compartment. ${ }^{13}$ In a sitting position, it was found that the CSF-containing lumbar subarachnoid space had a pressure of $0 \mathrm{mmHg}$ at the level of the occiput of the skull, a height similar to the globe. Thus, the pressure around the optic nerve is likely less than that measured with lumbar puncture performed in a lateral decubitus position. An associated issue is that IOP has also been found to vary with posture. ${ }^{14}$ The IOP increases when moving from an upright to horizontal position. Furthermore, such an IOP increase is greater in those with glaucoma over normal controls. ${ }^{15}$ One study demonstrated a $2.9 \mathrm{~mm} \mathrm{Hg}$ increase from a seated to supine position change in healthy controls and a $3.9 \mathrm{~mm} \mathrm{Hg}$ change in those with glaucoma. ${ }^{5}$ The current explanation for IOP variation is that postural changes result in elevated episcleral venous pressure and choroidal congestion. ${ }^{17} 18$ In fact, an increase of episcleral venous pressure of $0.83 \pm 0.21 \mathrm{~mm} \mathrm{Hg}$ correlated with an increase of $1 \mathrm{~mm} \mathrm{Hg}$ in IOP. Several studies found that vascular congestion increased in inverted positions, placing pressure on ocular tissues and elevating IOP. Moreover, diurnal IOP fluctuations may affect the TLCPD gradient. ${ }^{19}$ However, research suggests that aqueous production is not affected by posture. ${ }^{20}$

Furthermore, recent investigations into CSF within the subarachnoid space surrounding the optic nerve suggest that there are variations in CSF flow surrounding the globe. ${ }^{21-24}$ The subarachnoid space encircling the optic nerve can be divided into three sections by the architecture of the trabeculae, septa and pillars that exist within the space. ${ }^{21}$ This divisive architecture could account for changes in CSF flow and even cause a 'compartment syndrome' in the subarachnoid space. ${ }^{23}$ This could result in variation in CSF-P at the lamina cribrosa and thus possibly alter TLCPD gradient. Compartmentalisation or decrease in CSF flow away from the optic nerve could also lead to accumulation of toxic metabolites or decreased nutrients.
Compartmentalisation of CSF around the optic nerve is evidenced by high concentration of lipocalin-like prostaglandin D-synthase (L-PGDS) found near the optic nerve head. L-PGDS is neuroprotective of astrocytes, modulates inflammation and is apoptotic and may alter the optic nerve and disease progression. Its presence simply confirms that the subarachnoid space of the optic nerve might not have free communication intracranial subarachnoid space and thus TLCPD might be accordingly affected. $^{23}$

\section{Early animal studies: IOP and CSF-P}

Early studies by Morgan et al in dogs provided some of the initial data on the relationship between IOP and CSF-P as well as retinal perfusion. In dogs, Morgan et al looked at the effect of CSF-P on retrolaminar tissue pressure and TLCPD at low CSF-P, thought to mimic that of the erect position. This study found that TLCPD is dependent on CSF-P when that pressure is above $0.5 \mathrm{~mm} \mathrm{Hg} .{ }^{8}$ In a later study, Morgan et al looked at the role of CSF-P on glaucoma. This study found that CSF-P and IOP have equivalent effects on TLCPD and optic disc surface movement. It was also found that CSF-P affects axonal transport of the optic nerve, which might have an effect on glaucoma aetiology and retinal venous outflow. ${ }^{25}$

Recently, there has been enhanced understanding of the retinal vein pulsation by Morgan et $a l^{26}$, who determined that the central retinal vein collapse in the eye occurs in conjunction with intraocular diastole, not systole. It also occurs in time with IOP and ICP diastole. Furthermore, the central retinal vein collapse occurred in an insignificant $0.6 \%$ of a cardiac cycle before the ICP minima but a significant $3.2 \%$ after the IOP minima, suggesting that ICP pulse pressure drives ocular venous pulsation. ${ }^{26}$ In general, the ICP phase occurs before the IOP phase, with ICP rising and falling slightly before IOP. The ICP pulse driving venous pulsation leads to increased outflow during the time of ICP minima. As the ICP rises first, leading to increased resistance to venous outflow, intraocular blood accumulates during systole. As the ICP falls slightly before the IOP in diastole, intraocular blood drains. Others have postulated that the phase difference would create a period of relatively higher CSF-P compared with IOP and promote retrograde axoplasmic flow of nutrients and metabolites towards the retina. ${ }^{27}$ Given the association of venous pulsation with ICP, it is hypothesised that there exists a low-resistance connection between the intracranial CSF and the CSF surrounding the optic nerve. ${ }^{26}$ This low-resistance connection does not have to be a continuous fluid compartment but can rather represent compartments produced by deformable septa that allow for pressure transfer.

\section{CSF-P, TLCPD and glaucoma}

In a study looking at individuals with high-tension glaucoma, normal tension glaucoma (NTG) and OHT, increased TLCPD gradient was negatively associated with neuroretinal rim area $(\mathrm{p}=0.006, \mathrm{r}=-0.38)$ and positively associated with mean visual field defect $(p=0.008, r=0.38){ }^{28}$ Berdahl and colleagues observed that cup-to-disc ratio was positively correlated with TLCPD gradient. ${ }^{29}$ They also found that CSF-P was 33\% lower in study participants with primary open angle glaucoma (POAG) compared with non-glaucomatous controls $(9.2 \mathrm{~mm} \mathrm{Hg}$ vs $13.0 \mathrm{~mm} \mathrm{Hg}$ ).

If indeed TLCPD is associated with glaucoma, one might expect NTG patients to have reduced CSF-P causing increased gradient despite normal IOP. In a prospective study by Ren et $a l^{10}$, CSF-P was significantly lower in the NTG group $(9.5 \mathrm{~mm} \mathrm{Hg})$ than both the high IOP glaucoma group 
$(11.7 \mathrm{~mm} \mathrm{Hg})$ and the control group (12.9 $\mathrm{mm} \mathrm{Hg})$. The TLCPD gradient was significantly higher in those with glaucoma (NTG $6.6 \mathrm{~mm} \mathrm{Hg}$, high IOP glaucoma $12.5 \mathrm{~mm} \mathrm{Hg}$ ) compared with controls $(1.4 \mathrm{~mm} \mathrm{Hg})$. Using multivariate analysis, the TLCPD gradient was significantly associated with perimetric visual field loss. However, when IOP and CSF-P were used as individual parameters in the multivariate analysis, there was no significant correlation between these individual parameters and glaucomatous visual field loss. ${ }^{10}$

Perhaps increased CSF-P in OHT patients lowers TLCPD gradient and explains the lack of glaucomatous damage despite elevated IOP. When comparing OHT patients with nonglaucoma controls, Ren et $a l^{30}$ found OHT patients to have significantly higher CSF-P than controls $(16.0 \pm 2.5 \mathrm{~mm} \mathrm{Hg}$ and $12.9 \pm 1.9 \mathrm{~mm} \mathrm{Hg}$, respectively). Berdahl et $a^{29}$ also found significantly higher CSF-P in OHT patients over controls. However, despite the association of higher CSF-P in OHT patients with higher IOP, TLCPD gradient was significantly higher in the OHT group. ${ }^{30}$ Ren and colleagues hypothesised this could be explained by a pre-glaucomatous state in which detectable perimetric or morphological changes had not yet developed. They noted that patients in this group, if in fact they would eventually develop glaucomatous damage, could have benefitted from antiglaucoma treatment. ${ }^{30}$ Perhaps there is a threshold pressure gradient to pass to develop damage, and such a gradient is surpassed in OAG but not OHT. In fact, a previous study showed that OHT patients have a lower TLCPD gradient than POAG patients. ${ }^{9}$

\section{BP, IOP, CSF-P AND OAG RISK}

Large population-based studies have determined that IOP is significantly associated with both systolic BP (SBP) and diastolic BP (DBP). ${ }^{31-36}$ Dielemans et $a l^{34}$ demonstrated that a $10 \mathrm{~mm} \mathrm{Hg}$ increase in SBP resulted in a $0.23 \mathrm{~mm} \mathrm{Hg}$ increase in IOP, and a $10 \mathrm{~mm} \mathrm{Hg}$ increase in DBP caused a $0.24 \mathrm{~mm} \mathrm{Hg}$ IOP increase. Similarly, data from the Egna-Neumarkt Study found that $10 \mathrm{~mm} \mathrm{Hg}$ increments in SBP and DBP caused IOP increases of 0.24 and 0.40 , respectively. ${ }^{36}$

However, more notable is the extensive literature that points towards the effect of decreased BP on OAG risk. A DBP less than $90 \mathrm{~mm} \mathrm{Hg}$ due to antihypertensive therapy in nonglaucoma eyes is associated with decreased rim area and increased optic disc cupping. ${ }^{37}$ Furthermore, various studies have demonstrated a relationship between glaucoma progression and hypotension. ${ }^{38} 39$ Patients with OAG with progression despite well-controlled IOP, as well as NTG patients, exhibited lower SBP throughout the day and night compared with healthy controls. $^{28}$ Patients with uncontrolled IOP did not have a BP that varied from the controls. Subsequently, Graham et $a l^{39}$ found that a higher risk for glaucoma progression was associated with large decreases in nocturnal BP. ${ }^{40}$ With this in mind, some argue that underlying impaired autoregulation of ocular blood flow causes ischaemic periods followed by reperfusion injury. In accordance, increased mean ocular perfusion pressure fluctuation has been implicated as a risk factor for glaucoma severity in NTG patients. ${ }^{40}$ Thus, appropriate SBP control is imperative in glaucoma treatment. However, reduction of arterial hypertension will also reduce CSF-P and affect the TLCPD gradient.

The correlation between decreased BP and OAG risk could be explained by the relationship between CSF-P and BP. It has been suggested that reduction of arterial $\mathrm{BP}$ has been associated with greater decrease in CSF-P than IOP in NTG patients. ${ }^{41}$ Disparate pressure decrease would increase the TLCPD gradient and could explain glaucomatous optic nerve changes in patients with normal IOP. Conversely, increased SBP may be associated with increased CSF-P and may thus protect against glaucomatous damage in $\mathrm{OHT}^{41}$ Interestingly, arterial hypertension and elevated CSF-P have also been implicated as risk factors for retinal vein occlusion. ${ }^{41}$ Data on this topic are limited, and more research is needed.

\section{CARBONIC ANHYDRASE INHIBITORS}

Currently, the only therapeutic strategies available to treat OAG are targeted at lowering IOP. The four classes of topical medications include prostaglandin analogues, $\beta$-adrenergic receptor antagonists, $\alpha 2$-adrenergic receptor agonists and carbonic anhydrase inhibitors. It is important to discuss the use of carbonic anhydrase inhibitors specifically as they are also known to decrease CSF production. ${ }^{42}$ Carbonic anhydrase inhibitors remain an effective glaucoma treatment modality by lowering IOP through reduction of aqueous humour formation. ${ }^{43}$ They have been used in topical forms for POAG and in systemic forms for acutely elevated IOP. ${ }^{1}$ Additionally, carbonic anhydrase inhibitors are used to decrease CSF production and thus lower CSF-P in patients with idiopathic intracranial hypertension. ${ }^{42}$ With increasing evidence that TLCPD is associated with glaucoma progression, carbonic anhydrase inhibitor effects on IOP and CSF-P should be further evaluated. It is expected that systemic carbonic anhydrase inhibitor administration for acute angle closure glaucoma will reduce both IOP and CSF-P. As previously discussed, only TLCPD gradient, and neither IOP nor CSF-P alone, was significantly associated with perimetric loss. ${ }^{10}$ Data are currently lacking as to how this class of medications affects the interplay between IOP and CSF-P in glaucoma, and no conclusions can be made at this time.

\section{Relationship between BMI, IOP and CSF-P}

BMI has been shown to be positively correlated with IOP with multivariate regression analysis, ${ }^{44-47}$ even in children. ${ }^{48}$ Some postulate that increased adipose tissue fills the orbit and increases episcleral venous pressure, raising $\mathrm{IOP}^{49}$ Others state lipid deposits could reduce aqueous fluid outflow. ${ }^{50}$ Recently, a study found that BMI only had a statistically significant association with IOP when insulin resistance data were removed as a covariate. ${ }^{51}$ It was suggested that perhaps the BMI and IOP relationship may be a manifestation of an association between insulin resistance and IOP.

However, some studies did not reach statistical significance. For instance, several studies only found a positive correlation between IOP and BMI in men, not women. ${ }^{51} 52$ While the Beijing Study found a relationship using univariate analysis, no significant association was found through multivariate regression analysis. $^{35}$

Recently, BMI and its relation to glaucoma has been investigated. Pasquale et $a l^{53}$ demonstrated that in women increased BMI was associated with reduced risk of POAG with an IOP of 21 or less at diagnosis. No association was found in women with an IOP greater than 21 at diagnosis. No relationship was found in men. The Barbados Eye Study also concluded that OAG prevalence decreased with increasing BMI. ${ }^{54}$ Furthermore, men with a $B M I \geq 28.5$ had less than one-third the odds of having $\mathrm{OAG}$ of men with a $\mathrm{BMI} \leq 21.71$. Women with a $\mathrm{BMI} \geq 32.50$ had less than one-half the odds of OAG of women with a $\mathrm{BMI} \leq 23.44 .^{54}$ Perhaps racial differences can explain why Pasquale et al did not also find a correlation with men. While the Barbados Eye Study studied individuals of African descent, Pasquale et al investigated patients of European descent. ${ }^{52}$ 
Another explanation could be varying study methodology, as the Barbados Eye Study did not correct for central corneal thickness. However, Leske et al found that central corneal thickness was not correlated with BMI. ${ }^{55}$ In addition, the Singapore Malay Eye Study demonstrated reduced neuroretinal rim area and increased cup-to-disc ratio were associated with reduced BMI. ${ }^{56}$ In another population-based study, neuroretinal rim area was associated with increasing BMI. ${ }^{57}$ However, other studies suggest there is no correlation between BMI and glaucoma. ${ }^{58}$ Furthermore, a longitudinal cohort study of patients concluded that OAG is more common in obesity with a HR of $1.06 .{ }^{59}$

If $\mathrm{BMI}$ is taken to reduce glaucoma risk, then the association of increasing CSF-P with increasing BMI, and subsequent effect on TLCPD gradient, might explain why this occurs. CSF-P has been shown to be linearly correlated with BMI. ${ }^{60}$ Another study found a trend between increased BMI and increased CSF-P but failed to reach statistical significance $(p=0.062) .{ }^{61}$ The pathophysiology behind the increase in CSF-P with increasing BMI is not fully understood, although theories have been postulated. One such theory states that CSF-P increase is caused by hypoventilation or obstructive sleep apnoea leading to respiratory acidosis. $^{50}$ Increased intra-abdominal pressure or venous outflow obstruction may also increase central venous pressure, resulting in increased CSF-P. ${ }^{62}$

Individuals with elevated BMI also are more likely to have diabetes mellitus and hypertension. ${ }^{53}$ Obesity, with its associated elevated CSF-P, appears protective for glaucoma; however, diabetes, which is also associated with obesity, is also considered a controversial risk factor for OAG. Several large-scale clinical trials have reported an increased risk of OAG in diabetics ${ }^{63-66}$ while other large trials have shown no such association. ${ }^{67-70}$ Despite this, it appears the protective effect of BMI against glaucoma overcomes the supposed increased risk of glaucoma due to diabetes mellitus and dysregulation ofBP. ${ }^{59}$ Perhaps obesity's association with hypertension can also partially explain the positive correlation between BMI and CSF-P. However, much remains to be explored in this relationship as other studies have failed to reach significance between CSF-P and obesity. ${ }^{61}$

\section{EFFECT OF AGE ON IOP AND CSF-P}

Age is a well-known risk factor for OAG. Population-based studies have found OAG prevalence to increase beginning in approximately the fifth decade of life. ${ }^{71} 72$ However, studies are inconsistent on the relationship between age and IOP, with some studies showing no correlation and other studies showing that IOP increases with age. ${ }^{73-75} \mathrm{~A}$ recent study by Fleischman et al found a significant correlation between age and CSF-P. This study found that CSF-P pressure began to decline steadily after age 50 with a $2.5 \%$ decrease in those aged 50-54 and a decrease of $26.9 \%$ in those aged 90-94 compared with those in the age group 29-40. ${ }^{76}$ These data suggest that TLCPD caused by a decreasing CSF-P with age might explain the increasing incidence of OAG with age.

\section{CONCLUSION}

In conclusion, regulation of pressures in the arterial system, the eye and the brain has profound influence on the development of glaucomatous change. Systemic arterial BP, IOP and CSF-P are inextricably tied to one another, and altering one often alters another. An increased TLCPD difference has been associated with visual field loss and increased neuroretinal rim area. This increased gradient, whether due to decreased CSF-P or due to increased IOP, may explain the existence of NTG and POAG, respectively. Conversely, a normal gradient, despite increased
CSF-P and IOP, may explain the lack of visible glaucomatous damage in OHT. Further research of TLCPD is warranted as some studies suggest OHT patients eventually develop optic nerve damage. Analysis of TLCPD gradients may help determine whether certain patients are more likely to develop glaucomatous damage or whether others are likely to progress more quickly with the disease. Better understanding of the IOP and CSF-P relationship could influence treatment regimens. Yet, as noted previously, the CSF-P and glaucoma relationship is complicated as CSF-P is affected by displacement of lamina cribrosa, body position, time of day and local changes in flow near the globe. Furthermore, with diabetes and hypertension on the rise, the BMI-CSF-P relationship warrants a front seat in the discussion of glaucoma. As tighter BP control in patients remains a high priority, the effects of changes in arterial BP on CSF-P should be considered. A better understanding of the relationships between such pressures and measurements has great potential in the treatment of glaucoma. Not unlike carbonic anhydrase inhibitors, glaucoma medications may influence more than one of these factors. Investigation of these factors may provide insight into disease progression in patients while on antiglaucoma medication.

Contributors All authors were involved in all aspects of the development of this manuscript.

Competing interests None.

Provenance and peer review Not commissioned; externally peer reviewed.

\section{REFERENCES}

1 Gerstenblith AT, Rabinowitz MP, Barahimi BI, et al. The wills eye manual: office and emergency room diagnosis and treatment of eye disease. Philadelphia: Lippincott, Williams and Wilkins, 2012

2 Leske MC. Open-angle glaucoma: an epidemiological overview. Ophthalmic Epidemiol 2007;14:166-72.

3 Quigley HA, Broman AT. The number of people with glaucoma worldwide in 2010 and 2020. Br J Ophthalmol 2006;90:262-7.

4 Murray CJ, Vos T, Lozano R, et al. Disability-adjusted life years (DALYs) for 291 diseases and injuries in 21 regions, 1990-2010: a systematic analysis for the Global Burden of Disease Study 2010. Lancet 2012;380:2197-223.

5 Vos T, Flaxman AD, Naghavi M, et al. Years lived with disability (YLDs) for 1160 sequelae of 289 diseases and injuries 1990-2010: a systematic analysis for the Global Burden of Disease Study 2010. Lancet 2012;380:2163-96.

6 Gamero GE, Fechtner RD. The optic nerve in glaucoma. In: Choplin NT, Lundy DC, eds. Atlas of glaucoma. 2nd edn. Andover: Informa, 2007:59-74.

7 Jonas J, Berenshtein E, Holbach L. Anatomic relationship between Lamina Cribrosa, Intraocular Space, and Cerebrospinal Fluid Space. Invest Ophthalmol Vis SCi 2003:44:5189-95.

8 Morgan WH, Yu DY, Alder VA, et al. The correlation between cerebrospinal fluid pressure and retrolaminar tissue pressure. Invest Opbthalmol Vis Set 1998;39: 1419-28.

9 Guidoboni G, Harris A, Carichino L, et al. Effect of Lamina cribrosa deformation on the Hemodynamics of the Central Retinal Artery: a mathematical model. Math Biosci Eng In press.

10 Ren $\mathrm{R}$, Jonas JB, Tian $\mathrm{G}$, et al. Cerebrospinal fluid pressure in glaucoma: a prospective study. Ophthalmology 2010;117:259-66.

11 Yablonski M, Ritch R, Pokorny KS. Effect of decreased intracranial pressure on optic disc. Invest Ophthalmol Vis Sci 1979;18:(Suppl):165.

12 Downs JC, Roberts MD, Burgoyne CF. Mechanical environment of the optic nerve head in glaucoma. Optom Vis Sci 2008;85:425-35.

13 Magnaes B. Body position and cerebrospinal fluid pressure. Part 2: clinical studies on orthostatic pressure and the hydrostatic indifference point. J Neurosurg 1976:44:698-705

14 Buys YM, Alashali T, Jin YP, et al. Effect of sleeping in a head-up position on intraocular pressure in patients with glaucoma. Opthalmology 2010;117:1348-51.

15 Jain MR, Marmion VJ. Rapid pneumatic and Machey-Marg applanation tonometry to evaluate the postural effect on intraocular pressure. $\mathrm{Br} J$ Ophthalmol 1976:60:687-93.

16 Krieglstein G, Langham ME. Influence of body position on the intraocular pressure of normal and glaucomatous eyes. Opthalmologica 1975;171:132-45.

17 Smith TJ, Lewis J. Effect of inverted body position intraocular pressure. Am J Ophthalmol 1985:99:617-18. 
18 Friberg TR, Sanborn G, Weinreb RN. Intraocular and episcleral venous pressure increase during inverted posture. Am J Ophthalmol 1987;103:523-6.

19 David R, Zangwill L, Briscoe D, et al. Diurnal intraocular pressure variations: an analysis of 690 diurnal curves. Br J Ophthalmol 1992;76:280-3.

20 Carlson $\mathrm{KH}$, McLaren JW, Topper JE, et al. Effect of body position on intraocular pressure and aqueous flow. Invest Ophthalmol Vis Sci 1987;28:1346-52.

21 Killer HE, Laeng HR, Flammer J, et al. Architecture of arachnoid trabeculae, pillars, and septa in the subarachnoid space of the human optic nerve: anatomy and clinical considerations. Br J Ophthalmol 2003;87:777-81.

22 Killer $\mathrm{HE}$, Jaggi GP, Flammer J, et al. The optic nerve: a new window into cerebrospinal fluid composition? Brain 2006;129:1027-30.

23 Killer HE, Jaggi GP, Flammer J, et al. Cerebrospinal fluid dynamics between the intracranial and the subarachnoid space of the optic nerve. Is it always bidirectional? Brain 2007;130:514-20.

24 Killer HE, Miller NR, Flammer J, et al. Cerebrospinal fluid exchange in the optic nerve in normal-tension glaucoma. Br J Ophthalmol 2012;96:544-8.

25 Morgan WH, Yu DY, Balaratnasingam C. The role of cerebrospinal fluid pressure in glaucoma pathophysiology: the dark side of the optic disc. J Glaucoma 2008;17:408-13.

26 Morgan WH, Lind CRP, Kain S, et al. Retinal vein pulsation is in phase with intracrania pressure and not intraocular pressure. Invest Ophthalmol Vis Sci 2012;53:4676-81.

27 Jonas JB, Wang N, Yang D. Retinal vein pulsation is in phase with intracranial pressure and not intraocular pressure. Invest Ophthalmol Vis Sci 2012;53:6045.

28 Ren R, Wang NL, Zhang $X$, et al. Trans-lamina cribrosa pressure difference correlated with neuroretinal rim area in glaucoma. Graefes Arch Clin Exp Ophthalmol 2011;249:1057-63.

29 Berdahl JP, Fautsch MP, Stinnett SS, et al. Intracranial pressure in primary open angle glaucoma, normal tension glaucoma, and ocular hypertension: a case-contro study. Invest Ophthalmol Vis Sci 2008:49:5412-18.

30 Ren $\mathrm{R}$, Zhang $\mathrm{X}$, Wang $\mathrm{N}$, et al. Cerebrospinal fluid pressure in ocular hypertension. Acta Ophthalmol 2011;89:142-8.

31 Mitchell P, Lee AJ, Wang JJ, et al. Intraocular pressure over the clinical range of blood pressure: blue mountains eye study findings. Am J Ophthalmol 2005;140:131-2.

32 Klein BEK, Klein R, Knudtson MD. Intraocular pressure and systemic blood pressure: longitudinal perspective: the Beaver Dam Eye Study. Br J Ophthalmol 2005:89:284-7.

33 Tomoyose E, Higa A, Sakai $H$, et al. Intraocular pressure and related systemic and ocular biometric factors in a population-based study in Japan: the Kumejima study. Am J Ophthalmol 2010;150:279-86.

34 Dielemans I, Vingerling JR, Algra D, et al. Primary open-angle glaucoma, intraocular pressure, and systemic blood pressure in the general elderly population. The Rotterdam Study. Ophthalmology 1995;102:54-60.

35 Xu L, Wang H, Wang Y, et al. Intraocular pressure correlated with arterial blood pressure: the Beijing eye study. Am J Ophthalmol 2007;144:461-2.

36 Bonomi L, Marchini G, Marraffa M, et al. Vascular risk factors for primary open angle glaucoma: the Egna-Neumarkt Study. Ophthalmology 2000;107:1287-93.

37 Topouzis F, Coleman AL, Harris A, et al. Association of blood pressure status with the optic disk structure in non-glaucoma subjects: the Thessaloniki eye study. Am J Ophthalmol 2006;142:60-7.

38 Kaiser HJ, Flammer J, Graf T, et al. Systemic blood pressure in glaucoma patients. Graefes Arch Clin Exp Ophthalmol 1993;231:677-80

39 Graham SL, Drance SM, Wijsman K, et al. Ambulatory blood pressure monitoring in glaucoma. The nocturnal dip. Ophthalmology 1995;102:61-9.

40 Choi J, Kim KH, Jeong J, et al. Circadian fluctuation of mean ocular perfusion pressure is a consistent risk factor for normal-tension glaucoma. Invest Ophthalmol Vis Sci 2007:48:104-11.

41 Jonas JB, Wang N. Association between arterial blood pressure, cerebrospinal fluid pressure and intraocular pressure in the pathophysiology of optic nerve head diseases. Clin Experiment Ophthalmol 2012:40:e233-4.

42 Samuels MA. Disturbances of Cerebrospinal Fluid and Its Circulation, Including Hydrocephalus, Pseudotumor Cerebri, and Low-Pressure Syndromes. In: Samuels MA, ed. Adams and Victor's principles of neurology. Chapter 30, 9th edn. New York: McGraw-Hill, 2009. http://www.accessmedicine.com/content.aspx? alD=3635067 (accessed 26 Nov 2013)

43 Flach AJ, Fraunfelder FW. Ophthalmic therapeutics. In: Riordan-Eva P, Cunningham ET Jr, eds. Vaughan \& Asbury's general ophthalmology. Chapter 22, 18th ed. New York: McGraw-Hill, 2011. http://www.accessmedicine.com/content. aspx?alD=55785305 (accessed 26 Nov 2013)

44 Klein BE, Klein R, Linton KL. Intraocular pressure in an American community. The Beaver Dam Eye Study. Invest Ophthalmol Vis Sci 1992:33:2224-8.

45 Mori $\mathrm{K}$, Ando F, Nomura $\mathrm{H}$, et al. Relationship between intraocular pressure and obesity in Japan. Int J Epidemiol 2000;29:661-6.
46 Wu SY, Leske MC. Associations with intraocular pressure in the Barbados Eye Study. Arch Ophthalmol 1997;115:1572-6.

47 Memarzadeh F, Ying-Lai M, Azen SP, et al.; Los Angeles Latino Eye Study Group. Associations with intraocular pressure in Latinos: the Los Angeles Latino Eye Study. Am J Ophthalmol 2008;146:69-76.

48 Akinci A, Cetinkaya E, Aycan Z, et al. Relationship between intraocular pressure and obesity in children. J Glaucoma 2007;16:627-30

49 Shiose $Y$, Kawase $Y$. A new approach to stratified normal intraocular pressure in a general population. Am J Ophthalmol 1986;101:714-21.

50 Bulpitt CJ, Hodes C, Everitt MG. Intraocular pressure and systemic blood pressure in the elderly. Br J Ophthalmol 1975;59:717-20.

51 Oh SW, Lee S, Park C, et al. Elevated intraocular pressure is associated with insulin resistance and metabolic syndrome. Diabetes Metab Rev 2005;21:434-40.

52 Yoshida M, Ishikawa M, Kokae A, et al. Association of life-style with intraocular pressure in middle-aged and older Japanese residents. Jpn J Ophthalmol 2003:47:191-8.

53 Pasquale LR, Willett WC, Rosner BA, et al. Anthropometric measures and their relation to incident primary open-angle glaucoma. Ophthalmology 2010;117:1521-9.

54 Leske MC, Connell AM, Wu SY, et al. Risk factors for open-angle glaucoma. The Barbados Eye Study. Arch Ophthalol 1995;113:918-24.

55 Casson RJ, Abraham LM, Newland HS, et al. Corneal thickness and intraocula pressure in a Nonglaucomatous Burmese population: the Meiktila Eye Study. Arch Ophthalmol 2008;126:981-5.

56 Zheng Y, Cheung CY, Wong TY, et al. Influence of height, weight, and body mass index on optic disc parameters. Invest Ophthalmol Vis Sci 2010;51:2998-3002.

$57 \mathrm{Xu}$ L, Wang YX, Wang S, et al. Neuroretinal rim area and body mass index. PLoS ONE 2012;7:e30104.

58 Gasser P, Stümpfig D, Schötzau A, et al. Body mass index in glaucoma. J Glaucoma 1999;8:8-11.

59 Newman-Casey PA, Talwar N, Nan B, et al. The relationship between components of metabolic syndrome and open-angle glaucoma. Ophthalmology 2011;118:1318-26.

60 Berdahl JP, Fleischman D, Zaydlarova J, et al. Body mass index has a linea relationship with cerebrospinal fluid pressure. Invest Ophthalmol Vis Sci 2012;53:1422-7.

61 Bono F, Lupo MR, Serra $P$, et al. Obesity does not induce abnormal CSF pressure in subjects with normal cerebral MR venography. Neurology 2002;59:1641-3.

62 Quattrone A, Gambardella A, Carbone AM, et al. A hypofibrinolytic state in overweight patients with cerebral venous thrombosis and isolated intracranial hypertension. J Neurol 1999;246:1086-9.

63 Klein BE, Klein R, Jensen SC. Open-angle glaucoma and older-onset diabetes. The Beaver Dam Eye Study. Ophthalmology 1994:101:1173-7.

64 Dielemans I, de Jong PT, Stolk R, et al. Primary open-angle glaucoma, intraocular pressure, and diabetes mellitus in the general elderly population. The Rotterdam Study. Ophthalmology 1996;103:1271-5.

65 Mitchell P, Smith W, Chey T, et al. Open angle glaucoma and diabetes: the Blue Mountains eye study, Australia. Ophthalmology 1997;104:712-8.

66 Chopra V, Varma R, Francis BA, et al. Type 2 diabetes mellitus and the risk of open-angle glaucoma the Los Angeles Latino Eye Study. Ophthalmology 2008;115:227-32.

67 Tielsch JM, Katz J, Quigley HA, et al. Diabetes, intraocular pressure, and primary open-angle glaucoma in the Baltimore Eye Survey. Ophthalmology 1995;102:48-53.

68 Gordon MO, Beiser JA, Kass MA. Is a history of diabetes mellitus protective against developing primary open-angle glaucoma? Arch Ophthalmol 2008;126:280-1.

69 de Voogd S, Ikram MK, Wolfs RC, et al. Is diabetes mellitus a risk factor for open-angle glaucoma? The Rotterdam Study. Ophthalmology 2006;113:1827-31.

70 Miglior S, Pfeiffer N, Torri V, et al. Predictive factors for open angle glaucoma among patients with ocular hypertension in the European Glaucoma Prevention Study. Ophthalmology 2007;114:3-9.

71 Klein BE, Klein R, Sponsel WE, et al. Prevalence of glaucoma: the Beaver Dam Eye Study. Ophthalmology 1992;99:1499-504.

72 Friedman DS; Eye Diseases Prevalence Research Group. Prevalence of open-angle glaucoma among adults in the United States. Arch Ophthalmol 2004;1224:532-8

73 Schulzer M, Drance SM. Intraocular pressure, systemic blood pressure, and age: a correlational study. BJO 1987;71:245-9

74 Nemesure B, Wu SY, Hennis A, et al. Factors related to the 4-year risk of high intraocular pressure: the Barbados Eye Studies. Arch Ophthalmol 2003;121:856-62.

75 Qureshi IA. Age and intraocular pressure: how are they correlated? JPMA 1995:45:150-2

76 Fleischman D, Berdahl J, Zaydlarova J, et al. Cerebrospinal fluid pressure decreases with older age. PLOS ONE 2012;7:e52664. 